\title{
Receiver Design for Noncoherent Digital Network Coding
}

\author{
Terry Ferrett*, Matthew C. Valenti*, and Don Torrieri ${ }^{\dagger}$ \\ ${ }^{*}$ West Virginia University, Morgantown, WV, USA. \\ $\dagger$ U.S. Army Research Laboratory, Adelphi, MD, USA.
}

\begin{abstract}
Physical-layer network coding is considered for the two-way relay network with realistic assumptions on the coherence of the channel. In contrast to analog network coding, which relays received analog signals plus noise, our system relays digital network codewords, obtained by digital demodulation and channel decoding. By using binary frequency-shift keying and noncoherent reception, the relay may operate without knowledge of the phases of the signals transmitted simultaneously by the two sources. The channels between the end nodes and the relay are modeled as noncoherent block fading channels, and an outer turbo code is used. A noncoherent receiver is formulated for the relay, which estimates the fading amplitudes but not the phases. Several block sizes are considered, and the effect of block size on error-rate performance is investigated. As a baseline for performance comparison, the system is also simulated using perfect knowledge of the fading amplitudes, and it is observed that the performance lost to channel estimation is negligible for sufficiently large blocks. An example realization of the proposed system demonstrates a $32.4 \%$ throughput improvement compared to a similar system that performs network coding at the link layer.
\end{abstract}

\section{INTRODUCTION}

Network coding is a relaying technique that increases throughput over traditional store-and-forward relaying [1]. The two-way relay channel (TWRC) is the most fundamental topology that can exploit network coding techniques [2]. The TWRC is a three-terminal network consisting of a pair of source nodes $\mathcal{N}_{1}$ and $\mathcal{N}_{2}$ that exchange information via a single relay node $\mathcal{R}$. Information is exchanged, making $\mathcal{N}_{j}$ the destination of node $\mathcal{N}_{i}, i \neq j$. In this topology, network coding can be applied at either the link layer or the physical layer [3]. Information may be protected by using an errorcorrecting channel code, which is applied either on a linkby-link basis or on an end-to-end basis [4]. Using link-bylink channel coding, both the end nodes and the relay apply channel codes to the data. The channel codes applied by the end nodes and the relay may be different. Using end-to-end coding, only nodes $\mathcal{N}_{1}$ and $\mathcal{N}_{2}$ perform channel decoding, not the relay. Because the decoding operation at the relay minimizes error propagation and noise accumulation over the two links, link-by-link channel coding offers potentially better performance than end-to-end coding at the cost of increased complexity.

In Fig. 1, link-layer network coding (LNC) is compared against physical-layer network coding (PNC) in the channelcoded TWRC. Let $\mathbf{u}_{i}$ indicate the message of source node $\mathcal{N}_{i}$. The modulated and channel-coded signal transmitted by $\mathcal{N}_{i}$ is denoted by $\Gamma_{S}\left(\mathbf{u}_{i}\right)$. With LNC, which is shown in Fig. 1(a), nodes $\mathcal{N}_{1}$ and $\mathcal{N}_{2}$ transmit their signals during two disjoint time slots. Using link-by-link channel coding, the two signals are demodulated and channel-decoded at the relay to obtain estimates $\hat{\mathbf{u}}_{1}$ and $\hat{\mathbf{u}}_{2}$. Assuming the network code is defined over finite field GF(2), a network codeword $\mathbf{u}=\hat{\mathbf{u}}_{1} \oplus \hat{\mathbf{u}}_{2}$ is

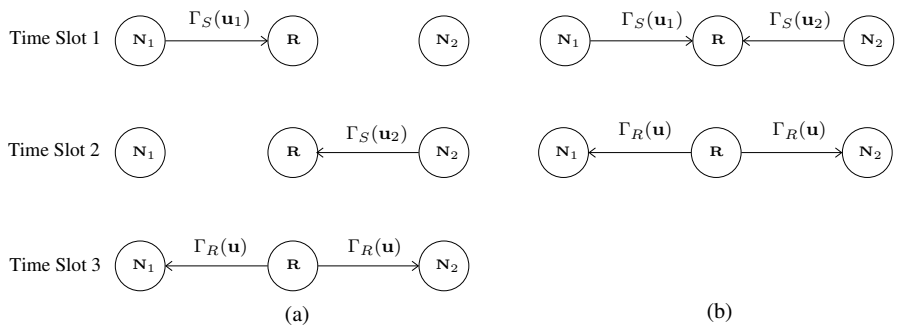

Fig. 1. (a) Link-layer network coding, and (b) Physical-layer network coding.

formed by taking the modulo-two sum of the two estimated codewords. During a third time slot, the relay broadcasts signal $\Gamma_{R}(\mathbf{u})$, which is a modulated and channel-coded version of $\mathbf{u}$, and the two destination nodes demodulate and decode the received versions of this signal, revealing estimates of $\mathbf{u}$. Finally, node $\mathcal{N}_{j}$ recovers an estimate $\tilde{\mathbf{u}}_{i}$ of source node $\mathcal{N}_{i}$ 's transmission, $i \neq j$, by adding its own information to its estimate $\hat{\mathbf{u}}$ of the network codeword, i.e. $\tilde{\mathbf{u}}_{i}=\hat{\mathbf{u}} \oplus \mathbf{u}_{j}$.

As shown in Fig. 1(b), PNC reduces the number of time slots required for transmission to two. The source nodes transmit their signals over a multiple-access channel (MAC) in the same time slot. The PNC system does not detect the messages $\mathbf{u}_{1}$ and $\mathbf{u}_{2}$ separately, as in LNC. Instead, the network-coded message $\mathbf{u}$ is detected directly by the relay's demodulator from the electromagnetic sum of received signals. As with the LNC system, each source node obtains an estimate of the opposite source node's message by adding its own information to its estimate $\hat{\mathbf{u}}$ of the network codeword.

The mapping from received signal to transmitted signal at the relay is critical. One option is for the relay to simply retransmit the signal it receives, with no demodulation or decoding. This approach was proposed by Katti et al. in [5], which calls the scheme analog network coding (ANC). A refinement to ANC, which reduces power consumption at the relay by using a Tomlinson-Harashima precoding, is presented by Hao et. al. [6], which refers to the TWRC channel as the exchange channel. With ANC, the network codeword $\mathbf{u}$ at the relay is not formed using hard decisions over a finite field, but rather is defined over an infinite field. For this reason, Zhang et al. in [7] offer the alternative term PNC over an infinite field (PNCI) to describe ANC. The main disadvantage of ANC is that noise received by the relay is amplified, and added to additional noise during the broadcast step. Furthermore, because channel decoding is not performed at the relay, ANC systems cannot use link-by-link channel coding.

An alternative to nonregenerative signal forwarding is to demodulate and decode the signal at the relay to obtain the network codeword $\mathbf{u}$, which is then re-encoded and remodulated. In this case, the decoding operation assures that the network codeword is defined over a finite field (usually the same field as the channel code). In [7], this scheme is referred to as PNC over a finite field (PNCF). In this paper, we choose to use the alternative term digital network coding (DNC) 


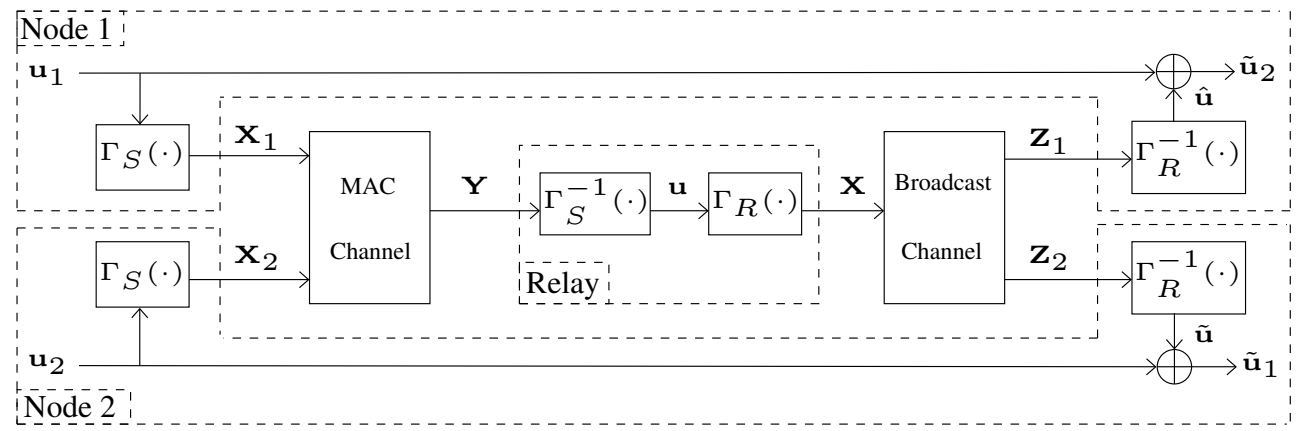

Fig. 2. Discrete-time system model.

to emphasize the difference with ANC. Unlike ANC, DNC removes the noise at the relay. As one of the two signals at the relay becomes weaker than the other, ANC's performance for the weaker signal degenerates. However, for DNC, the loss is relatively minor for both signals.

Symbol timing is an important consideration in all PNC systems. Assume that the clocks at the two source nodes are perfectly synchronized. This synchronization can be achieved by periodically updating the network timing at all the nodes and requiring that packet transmissions begin during defined time slots. Such a process is routine in cellular and other networks with scheduling mechanisms. Despite the common clocks at the source nodes, a misalignment or timing offset occurs when the propagation times of the two signals differ. The offset is $\tau=\Delta_{d} / c$, where $\Delta_{d}$ is the difference in link distances from the two sources to the relay and $c$ is the speed of light. For this effect to be small, we need $\tau<<T_{s} / 2$, where $T_{s}$ is the symbol period. Thus, the symbol rate is limited. For example, if $\Delta_{d}=300 \mathrm{~m}$, then $T_{s}>>2$ microsec is needed, and it is desirable for the symbol rate to be no more than 250 $\mathrm{ksym} / \mathrm{s}$. The system can compensate for the misalignment by delaying the transmission of the closer node by $\tau$, but this requires the distances to be tracked, and the change in delay may have other repercussions on the network.

Under the assumptions of perfect power control and phase synchronization, Zhang et al. [3] derive the bit error probability at the relay for coherent binary phase-shift keying. The phase synchronization assumption requires the relative carrierphase offset of the two signals to be available to the receiver. This requires very stable oscillators, an assumption not present in our work. There is also an additional throughput loss and overhead cost because measuring the carrier-phase offset requires the transmission of two non-overlapping preambles. Thus, coherent DNC is not practical, and a practical DNC system should use noncoherent demodulation at the relay.

A turbo-coded DNC system which uses orthogonal CPFSK modulation and a noncoherent relay receiver is presented in [8]. While the relay receiver does not require knowledge of the absolute received phases of the two signals, it is able to exploit other types of channel state information (CSI), such as the received amplitudes. By properly using CSI, a performance gain of approximately $10 \mathrm{~dB}$ may be achieved relative to a receiver that does not use CSI. This large performance improvement is motivation for carefully considering the issue of fading amplitude estimation, which was not considered in [8]. A training-based approach to channel estimation for coherent ANC systems is considered in [9], but such an approach decreases throughput, and hence is not desirable.

In this paper, we adapt an estimator originally proposed by Hamkins [10] for use in the noncoherent DNC system. We assume that the phase differences of the two signals received at the relay may vary rapidly while the fading amplitudes remain constant over a fading block. Oscillator instabilities lead to this type of distortion, which may be mitigated by using noncoherent FSK [11]. We further assume that symbollevel synchronization is achieved at the relay with negligible misalignment. Simulation results are presented for a turbocoded system operating over a Rayleigh block-fading channel. It is shown by example that the proposed DNC system is capable of achieving a $32.4 \%$ higher throughput than an equivalent LNC system.

\section{SySTEM MODEL}

The discrete-time system model shown in Fig. 2 gives an overview of the processing at all three nodes. Source node $\mathcal{N}_{i}, i \in\{1,2\}$, generates a length- $K$ information sequence, $\mathbf{u}_{i}=\left[u_{i, 1}, \ldots, u_{i, K}\right]$. The source nodes channelencode and modulate their information sequences using the function $\Gamma_{S}(\cdot)$, which is common to both nodes. A rate- $r_{S}$ turbo code is used, and the resulting length $L_{S}=K / r_{S}$ turbo codeword generated by $\mathcal{N}_{i}$ is denoted by $\mathbf{b}_{i}=\left[b_{i, 1}, \ldots b_{i, L_{S}}\right]$ (not shown in the diagram). The signal transmitted by node $\mathcal{N}_{i}$ during signaling interval $k T_{s} \leq t \leq(k+1) T_{s}$ is

$$
s_{i}(t)=\sqrt{\frac{2}{T_{s}}} \cos \left[2 \pi\left(f_{c_{i}}+\frac{b_{i, k}}{T_{s}}\right)\left(t-k T_{s}\right)\right]
$$

where $f_{c_{i}}$ is the carrier frequency of node $\mathcal{N}_{i}$ (not assumed to be exactly the same for both nodes) and $T_{s}$ is the symbol period. Note that (1) is continuous-phase frequency-shift keying (CPFSK) with a unity modulation index, which is orthogonal under noncoherent demodulation and has a continuous phase transition from one symbol to the next [12]. The orthogonallymodulated signal $s_{i}(t)$ may be represented in discrete time by the $2 \times L_{S}$ matrix $\mathbf{X}_{i}=\left[\mathbf{x}_{i, 1}, \ldots, \mathbf{x}_{i, L_{S}}\right]$ with $k^{t h}$ column

$$
\mathbf{x}_{i, k}= \begin{cases}{\left[\begin{array}{ll}
1 & 0
\end{array}\right]^{T}} & \text { if } b_{i, k}=0 \\
{\left[\begin{array}{ll}
0 & 1
\end{array}\right]^{T}} & \text { if } b_{i, k}=1 .\end{cases}
$$

For the DNC system, the signals are transmitted simultaneously by the two source nodes over a MAC channel. The relay receives the noisy electromagnetic sum of interfered and faded signals, $\mathbf{Y}$, and applies the demodulation and channeldecoding function $\Gamma_{S}^{-1}(\cdot)$. The demodulation operation yields a soft estimate of the network-and-channel-coded message $\mathbf{b}=\mathbf{b}_{1} \oplus \mathbf{b}_{2}$ (not shown), while the channel-decoding operation yields a hard-decision on the network-coded message $\mathbf{u}=\mathbf{u}_{1} \oplus \mathbf{u}_{2}$. With the LNC system, the two sources transmit during orthogonal time slots. The received versions of $\mathbf{X}_{1}$ and $\mathbf{X}_{2}$ are demodulated independently to provide soft estimates of $\mathbf{b}_{1}$ and $\mathbf{b}_{2}$. These soft estimates are combined and turbo decoded to yield a hard estimate of $\mathbf{u}$. The key distinction between DNC and LNC is that with the DNC system, the estimate of $\mathbf{b}$ is obtained directly from $\mathbf{Y}$, while with LNC it is found by independently demodulating the two source signals and then combining them. See [8] for more detail regarding this distinction. 
During the broadcast phase, the relay encodes and modulates $\mathbf{u}$ using the function $\Gamma_{R}(\cdot)$, which may be different than the function $\Gamma_{S}(\cdot)$ used by the sources. The channel code applied by the relay is a rate $r_{R}$ turbo code, yielding a length $L_{R}=K / r_{R}$ turbo codeword. While the MAC channel in a DNC system is subject to interference, the broadcast channel is not. For this reason, the code for the MAC channel must typically be stronger than the one for the broadcast channel, and it is desirable to select $r_{S}<r_{R}$. The relay broadcasts its encoded and modulated signal, which may be represented in discrete-time by the $2 \times L_{R}$ matrix $\mathbf{X}$. The signal traverses two independent fading channels, and the end nodes receive independently faded versions of $\mathbf{X}: \mathbf{Z}_{1}$ at $\mathcal{N}_{1}$ and $\mathbf{Z}_{2}$ at $\mathcal{N}_{2}$. The end nodes demodulate and decode their received signals using the function $\Gamma_{R}^{-1}(\cdot)$, and form estimates of $\mathbf{u}$. Let $\hat{\mathbf{u}}$ denote the estimate at $\mathcal{N}_{1}$ and $\tilde{\mathbf{u}}$ denote the estimate at $\mathcal{N}_{2}$. Next, estimates of the transmitted information messages are formed, $\tilde{\mathbf{u}}_{2}=\hat{\mathbf{u}} \oplus \mathbf{u}_{1}$ at $\mathcal{N}_{1}$ and $\tilde{\mathbf{u}}_{1}=\tilde{\mathbf{u}} \oplus \mathbf{u}_{2}$ at $\mathcal{N}_{2}$. Since the links in the broadcast phase are conventional point-topoint links with no interference, specific details of the receiver formulation will not be presented here. A detailed exposition of receiver design for turbo-coded CPFSK systems in block fading channels can be found in [13].

All of the channels in the system are modeled as blockfading channels. A block is defined as a set of $N$ symbols that all experience the same fading amplitude. The duration of each block corresponds roughly to the channel coherence time. Ideally both sources transmit with the same carrier frequency, i.e $f_{c_{1}}=f_{c_{2}}$. However, due to instabilities in the source node's oscillators and different Doppler shifts due to independent motion, it is not feasible to assume that these two frequencies are the same at the relay receiver. At best, the relay receiver could lock onto one of the two frequencies, in which case the received phase of the other signal would drift from one symbol to the next. To model this behavior, we let the phase shift of each symbol within a block vary independently.

The signal matrix $\mathbf{X}_{i}$ transmitted by node $\mathcal{N}_{i}$ may be partitioned into $N_{b}=L_{S} / N$ blocks according to

$$
\mathbf{X}_{i}=\left[\begin{array}{lll}
\mathbf{X}_{i}^{(1)} & \ldots & \mathbf{X}_{i}^{\left(N_{b}\right)}
\end{array}\right]
$$

where each block $\mathbf{X}_{i}^{(\ell)}, 1 \leq \ell \leq N_{b}$, is a $2 \times N$ matrix, and $N_{b}$ is assumed to be an integer. The channel associated with block $\mathbf{X}_{i}^{(\ell)}$ is represented by the $2 \times N$ diagonal matrix $\mathbf{H}_{i}^{(\ell)}=\alpha_{i}^{(\ell)} \times \operatorname{diag}\left(\exp \left\{j \theta_{i, 1}^{(\ell)}\right\}, \ldots, \exp \left\{j \theta_{i, N}^{(\ell)}\right\}\right)$, where $\alpha_{i}^{(\ell)}$ is a real-valued fading amplitude and $\theta_{i, k}^{(\ell)}$ is the phase shift of the $k^{\text {th }}$ symbol. The $\theta_{i, k}^{(\ell)}$, s are independent and identically distributed over $U(0,2 \pi)$. The fading amplitudes take into account not only the small-scale fading, but also the path loss and transmit power. The $\ell^{t h}$ block at the sampled output of the relay receiver's matched-filters is then

$$
\mathbf{Y}^{(\ell)}=\mathbf{X}_{1}^{(\ell)} \mathbf{H}_{1}^{(\ell)}+\mathbf{X}_{2}^{(\ell)} \mathbf{H}_{2}^{(\ell)}+\mathbf{N}^{(\ell)}
$$

where $\mathbf{N}^{(\ell)}$ is a $2 \times N$ noise matrix whose elements are i.i.d. circularly-symmetric complex Gaussian random variables with zero mean and variance $N_{0}$.

\section{RELAY RECEIVER}

At the relay, each block $\mathbf{Y}^{(\ell)}$ of the channel observation matrix $\mathbf{Y}$ is passed to a channel estimator, which computes estimates of the $\alpha_{1}^{(\ell)}$ and $\alpha_{2}^{(\ell)}$. A full description of the estimator is given in Section IV. The fading amplitude estimates and channel observations are used to obtain soft-estimates of the network-and-channel-coded sequence b. A full description and derivation of the relay receiver can be found in [8], so we will only provide an overview of the receiver in this Section.

The demodulator operates on a symbol-by-symbol basis, and therefore we may focus on a single signaling interval by dropping the dependence on the symbol interval $k$ and the block index $\ell$. Let $b_{1}$ and $b_{2}$ be the turbo-coded bits transmitted by nodes $\mathcal{N}_{1}$ and $\mathcal{N}_{2}$, and let $b=b_{1} \oplus b_{2}$ be the corresponding network-coded bit. The relay demodulator computes the loglikelihood ratio (LLR)

$$
\Lambda(b)=\log \frac{P(b=1 \mid \mathbf{y})}{P(b=0 \mid \mathbf{y})}=\log \frac{P\left(b_{1} \oplus b_{2}=1 \mid \mathbf{y}\right)}{P\left(b_{1} \oplus b_{2}=0 \mid \mathbf{y}\right)}
$$

where $\mathbf{y}$ is the corresponding column of $\mathbf{Y}$.

Define the following four mutually-exlusive events:

1) Event $\mathbb{E}_{1}=\left\{b_{1}=0, b_{2}=0\right\}$

2) Event $\mathbb{E}_{2}=\left\{b_{1}=1, b_{2}=1\right\}$

3) Event $\mathbb{E}_{3}=\left\{b_{1}=0, b_{2}=1\right\}$

4) Event $\mathbb{E}_{4}=\left\{b_{1}=1, b_{2}=0\right\}$.

The LLR in (5) can be represented in terms of these events according to

$$
\Lambda(b)=\log \frac{P\left(\mathbb{E}_{3} \mid \mathbf{y}\right)+P\left(\mathbb{E}_{4} \mid \mathbf{y}\right)}{P\left(\mathbb{E}_{1} \mid \mathbf{y}\right)+P\left(\mathbb{E}_{2} \mid \mathbf{y}\right)}
$$

Let $\alpha_{1}$ and $\alpha_{2}$ be the fading amplitudes over the two channels during this signaling interval, and let $\theta_{1}$ and $\theta_{2}$ be the received phases. Define $\alpha=\left|\alpha_{1} \exp \left(j \theta_{1}\right)+\alpha_{2} \exp \left(j \theta_{2}\right)\right|=$ $\left[\alpha_{1}^{2}+2 \alpha_{1} \alpha_{2} \cos (\theta)+\alpha_{2}^{2}\right]^{1 / 2}$, where $\theta=\theta_{2}-\theta_{1}$ is the difference between the two received phases. Since it is the magnitude of the sum of two zero-mean circularly-symmetric complex Gaussian variables, it follows that $\alpha$ is Rayleigh. The phases vary independently for every symbol in each fading block, so estimating $\alpha$ is not practical. Instead, $\alpha^{2}$ is approximated using its mean value: $\alpha^{2} \approx \alpha_{1}^{2}+\alpha_{2}^{2}$. Using this approximation and Eq. (26) of [8], the LLR may be expressed, after some algebraic manipulation, as

$$
\begin{aligned}
\Lambda(b)= & \max *\left[F\left(\frac{2 \alpha_{1}\left|y_{1}\right|}{N_{0}}\right)+F\left(\frac{2 \alpha_{2}\left|y_{2}\right|}{N_{0}}\right),\right. \\
& \left.F\left(\frac{2 \alpha_{2}\left|y_{1}\right|}{N_{0}}\right)+F\left(\frac{2 \alpha_{1}\left|y_{2}\right|}{N_{0}}\right)\right] \\
& -\max *\left[F\left(\frac{2 \alpha\left|y_{1}\right|}{N_{0}}\right), F\left(\frac{2 \alpha\left|y_{2}\right|}{N_{0}}\right)\right]
\end{aligned}
$$

where $\max *(x, y)=\log \left(e^{x}+e^{y}\right), F(x)=\log \left[I_{0}(x)\right], I_{0}(x)$ is the zeroth-order modified Bessel function of the first kind, and the approximation $\alpha=\sqrt{\alpha_{1}^{2}+\alpha_{2}^{2}}$ is used.

\section{CHANNEL ESTIMATOR}

The channel estimator computes estimates $\hat{A}$ and $\hat{B}$ of the fading amplitudes $\alpha_{1}$ and $\alpha_{2}$. For a particular block of channel observations, $\mathbf{Y}_{\ell}$, the estimator first determines the matchedfilter values to use in computing the fading-amplitude estimates. The appropriate matched filter outputs are determined by the transmission-case detector, described in Subsection IV-A. Once the estimator has determined the matched-filter outputs to use for estimation, the fading amplitudes are estimated using the algorithm in Subsection IV-B. The fadingamplitude estimates are then passed to the demodulator. Since the form of the estimation algorithm is the same for each block, in the following discussion we can drop the dependence on $\ell$ and denote a block of received symbols by $\mathbf{Y}$.

Note that in expression (7), exchanging the values $\alpha_{1}$ and $\alpha_{2}$ does not change the final value of the expression. Expression (7) is commutative in $\alpha_{1}$ and $\alpha_{2}$. Therefore, the 
estimates $\hat{A}$ and $\hat{B}$ of the fading amplitudes can be assigned to $\alpha_{1}$ and $\alpha_{2}$ in either order. The relay estimator does not need to assign the fading-amplitude estimates to particular channels.

\section{A. Transmission-Case Detector}

The estimator must first determine the particular matchedfilter outputs to use in computing $\hat{A}$ and $\hat{B}$. Let $\mathbf{y}=\left[y_{1}, y_{2}\right]^{T}$ be a column in $\mathbf{Y}$ corresponding to a particular channel observation. It follows that $y_{1}$ is the matched-filter output corresponding to tone 1 , and $y_{2}$ is the matched-filter output corresponding to tone 2 , where tone 1 has frequency $f_{c}$, tone 2 has frequency $f_{c}+1 / T_{S}$, and we assume that $f_{c_{1}}=f_{c_{2}}=f_{c}$. For each observation $\mathbf{y}$, the estimator performs a hypothesis test to determine which matched-filter outputs to use:

1) Tone 1 transmitted by both sources. Use $y_{1}$.

2) Tone 2 transmitted by both sources. Use $y_{2}$.

3) Separate tones transmitted. Use sum of $y_{1}$ and $y_{2}$.

The hypothesis test is performed by choosing the most likely of the three events according to:

$$
\mathcal{H}=\underset{k \in\{1,2,3\}}{\operatorname{argmax}} g_{k}(\mathbf{y})
$$

where the functions $g_{k}(\mathbf{y})$ are conditional $\log$-likelihoods of the transmission cases, given $\mathbf{y}$. It follows that $\mathcal{H} \in\{1,2,3\}$.

Since the receiver does not yet have knowledge of the fading amplitudes, the estimator must compute the hypotheses without channel state information. The functions $g_{k}(\mathbf{y})$ are found by marginalizing Eq. (20) in [8] with respect to Eq. (30) in [8]. First, consider the two transmission events in which the end nodes transmit the same tone

$$
\begin{aligned}
g_{i}(\mathbf{y}) & =\log \left[P\left(\mathbb{E}_{i} \mid \mathbf{y}\right)\right] \\
& =\frac{\left|y_{i}\right|^{2}}{N_{0}\left(1+\frac{N_{0}}{\mathcal{E}_{1}+\mathcal{E}_{2}}\right)}-\log \left[1+\frac{\mathcal{E}_{1}+\mathcal{E}_{2}}{N_{0}}\right]
\end{aligned}
$$

where $i \in\{1,2\}$ and $\mathcal{E}_{i}=E\left[\left(\alpha_{i}^{(\ell)}\right)^{2}\right]$ is the energy of node $\mathcal{N}_{i}$ 's signal received at the relay, which is assumed to be known. Next, consider the transmission event for which the end nodes transmit separate tones

$$
\begin{aligned}
g_{3}(\mathbf{y})= & \log \left[P\left(\mathbb{E}_{3} \mid \mathbf{y}\right)+P\left(\mathbb{E}_{4} \mid \mathbf{y}\right)\right] \\
= & \log \left[\frac{\left(\mathcal{E}_{1} \mathcal{E}_{2}\right)^{2}}{\left(1+\frac{\mathcal{E}_{1}}{N_{0}}\right)\left(1+\frac{\mathcal{E}_{2}}{N_{0}}\right)}\right] \\
+ & \max *\left[\frac{\left|y_{1}\right|^{2}}{N_{0}\left(1+\frac{N_{0}}{\mathcal{E}_{1}}\right)}+\frac{\left|y_{2}\right|^{2}}{N_{0}\left(1+\frac{N_{0}}{\mathcal{E}_{2}}\right)},\right. \\
& \left.\frac{\left|y_{2}\right|^{2}}{N_{0}\left(1+\frac{N_{0}}{\mathcal{E}_{1}}\right)}+\frac{\left|y_{1}\right|^{2}}{N_{0}\left(1+\frac{N_{0}}{\mathcal{E}_{2}}\right)}\right] .
\end{aligned}
$$

Applying the hypothesis test to each channel observation in the block yields a sequence of $N$ test results, $\left\{\mathcal{H}_{1}, \ldots, \mathcal{H}_{N}\right\}$. Next, the receiver uses the hypothesis test results to extract the appropriate matched-filter outputs for every channel observation,

$$
v_{i}= \begin{cases}y_{1, i}, & \text { if } \mathcal{H}_{i}=1 \\ y_{2, i}, & \text { if } \mathcal{H}_{i}=2 \\ y_{1, i}+y_{2, i}, & \text { if } \mathcal{H}_{i}=3,\end{cases}
$$

which are placed into the vector $\mathbf{v}=\left[v_{1}, \ldots, v_{N}\right]$.

\section{B. DNC Fading-Amplitude Estimator}

The goal of the estimator is to determine estimates $\hat{A}$ and $\hat{B}$ of the magnitudes of $\alpha_{1}$ and $\alpha_{2}$ without respect to ordering. The estimator first computes a pair of averages, which are then used to determine the fading amplitudes. The averages computed by the estimator, and the quantities the averages are approximating, are [10]

$$
\begin{aligned}
X & =\frac{1}{N} \sum_{i=1}^{N}\left\|v_{i}\right\|^{2} \approx \alpha_{1}^{2}+\alpha_{2}^{2} \\
Y & =\frac{2}{N} \sum_{i:\left\|v_{i}\right\|^{2}>\beta}\left\|v_{i}\right\|^{2} \approx \alpha_{1}^{2}+\alpha_{2}^{2}+\frac{4 \alpha_{1} \alpha_{2}}{\pi}
\end{aligned}
$$

where $\beta$ is the median value of $\mathbf{v}$. Rearranging (12) yields the estimates $\hat{A}$ and $\hat{B}$ of the fading amplitudes

$$
\begin{aligned}
& \hat{A}=\frac{1}{2}\left(\sqrt{X+\frac{\pi}{2}(Y-X)}+\sqrt{X+\frac{\pi}{2}(X-Y)}\right) \\
& \hat{B}=\frac{1}{2}\left(\sqrt{X+\frac{\pi}{2}(Y-X)}-\sqrt{X+\frac{\pi}{2}(X-Y)}\right) .
\end{aligned}
$$

The estimator passes the fading amplitude estimates $\hat{A}$ and $\hat{B}$ to the demodulator, which assigns the estimates to the fading amplitudes $\alpha_{1}$ and $\alpha_{2}$ in the log-likelihood ratio (7) with no respect to order.

\section{Amplitude Estimation for Single-Transmitter Links}

During the broadcast phase, there is only a single transmission and the algorithm described in subsection IV-B cannot be used. Similarly, it cannot be used by the LNC system during the MAC phase since the two transmissions are over orthogonal channels. To estimate the fading amplitudes at the links involving only a single transmitter and receiver, the simple averaging technique given by (29) in [14] is used, which is described as follows. Consider the $i^{\text {th }}$ signaling interval during the $\ell^{\text {th }}$ fading block. Given transmission of tone $k$, in the absence of noise, the $k^{t h}$ matched-filter output at the receiver is $y_{k, i}=\alpha e^{j \theta_{i}}$, having magnitude $\left|y_{k, i}\right|=\alpha$. All other matched-filter outputs in the $i^{\text {th }}$ signaling interval are 0 . An estimate could be formed by taking the maximum $\left|y_{k, i}\right|$ over any column of $\mathbf{Y}_{\ell}$. In the presence of noise, an estimate of $\alpha$ can be formed by averaging across all columns of the fading block

$$
\hat{\alpha}=\frac{1}{N} \sum_{i=1}^{N} \max _{k}\left|y_{k, i}\right|
$$

\section{Simulation Study}

This section presents simulated performance results for the relay receiver described in Section III. The simulated link model is as described in Section II, with specific simulation parameters given in the following subsections. Unless otherwise specified, the DNC relay receiver uses the transmissioncase detector of Subsection IV-A and the fading-amplitude estimator of Subsection IV-B, while the LNC receiver and destination nodes (i.e. receivers in single-transmitter links) use (14). 


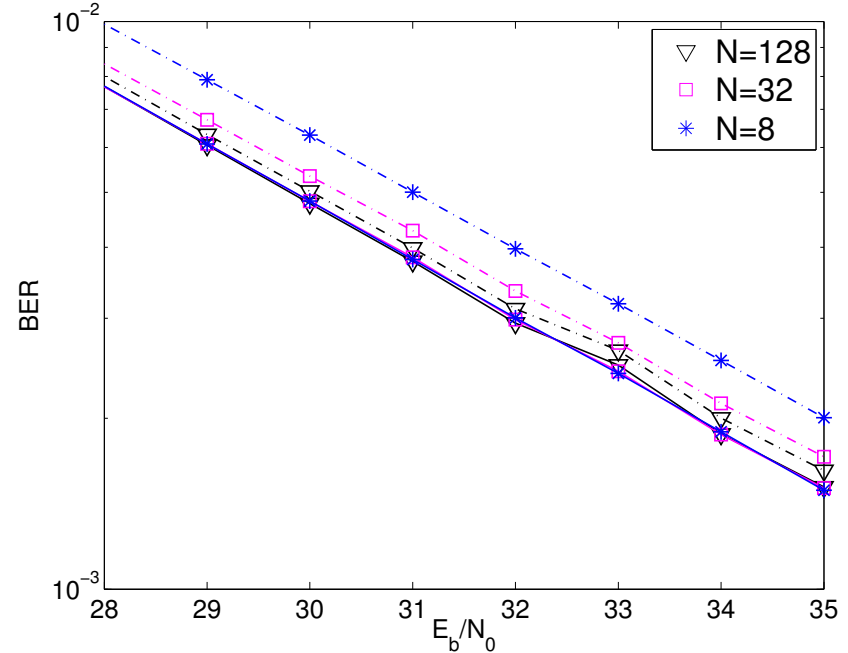

Fig. 3. Influence of fading-block length $N$ on uncoded DNC errorrate performance at the relay. Two curves are shown for each value of $N=\{8,32,128\}$. Solid curves - perfect fading-amplitude knowledge. Dashed curves - estimated fading amplitudes.

\section{A. Uncoded Relay Performance}

Consider a system that does not use an error-correcting code. The information frames generated at the end nodes contain $K=2048$ bits per frame. The fading blocks are length $N=\{8,32,128\}$ symbols per block. The DNC relay implements (7) and then makes a hard decision on each information bit.

The bit error-rate performance of the uncoded system is shown in Fig. 3. Two types of curves are shown in the figure, one representing performance with a perfect estimator, and the other using the estimator described in Section IV. A narrow range of BER's is shown to better highlight the differences in performance. In general, smaller fading blocks lead to a less accurate estimation of the fading amplitudes, as the number of samples available for estimation decreases. Moving from block size $N=128$ to 32 worsens performance by roughly $0.25 \mathrm{~dB}$, and from $N=32$ to 8 by $0.75 \mathrm{~dB}$.

\section{B. Performance with an Outer Turbo Code}

Now consider a system that uses an outer turbo code. The end nodes each encode length $K=1229$ information sequences into length $L=2048$ codewords, using a rate $r_{S} \approx 0.6$ UMTS turbo code [15]. The relay performs turbo decoding using the codeword LLR's computed by Eqn. (7). The fading-block lengths simulated are $N=\{8,16,32,64,128\}$ symbols per block.

The error performance of the coded system is shown in Fig. 4, both with perfect channel estimates and with estimated fading amplitudes. A good tradeoff between diversity and estimation accuracy is achieved at block size $N=16$, where the difference between the estimated and perfect-amplitude knowledge systems is about $0.7 \mathrm{~dB}$.

The SNR value required to reach an error rate of $10^{-4}$ in both systems is shown in Fig. 5 as a function of block length $N$. The performance of the LNC system degrades with increasing block size, as diversity is lost. With the DNC system, there is a tradeoff between diversity (short blocks) and estimator effectiveness (long blocks). The best performance for the DNC system is observed at $N=16$.

$\mathrm{An}$ error-rate performance comparison between DNC and LNC is shown in Fig. 6. Both systems use the same-rate turbo code. The LNC system outperforms the DNC system by

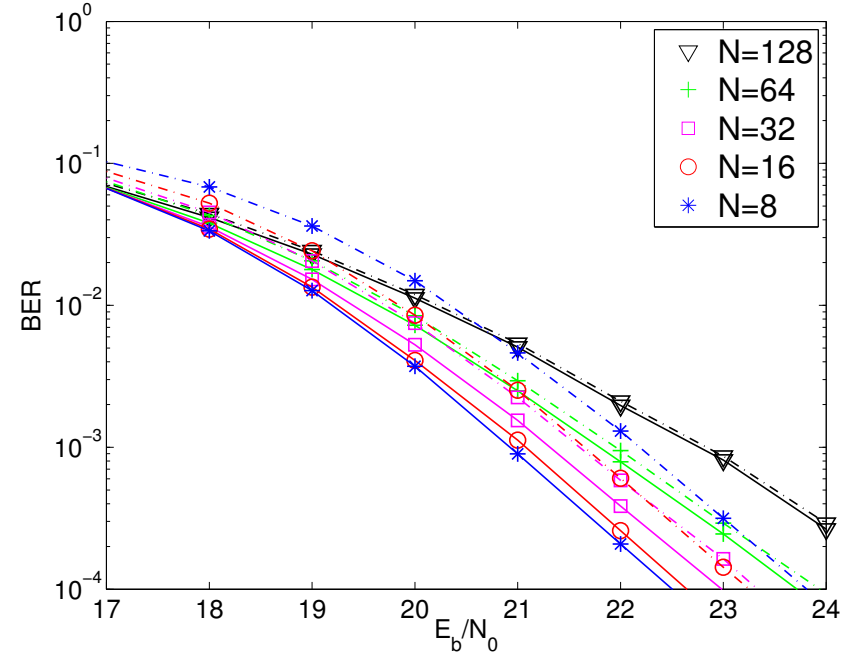

Fig. 4. Influence of fading-block length $N$ on turbo-coded DNC errorrate performance at the relay. Two curves are shown for each value of $N=\{8,16,32,64,128\}$. The solid curves - perfect fading-amplitude knowledge. Dashed curves - estimated fading amplitudes.

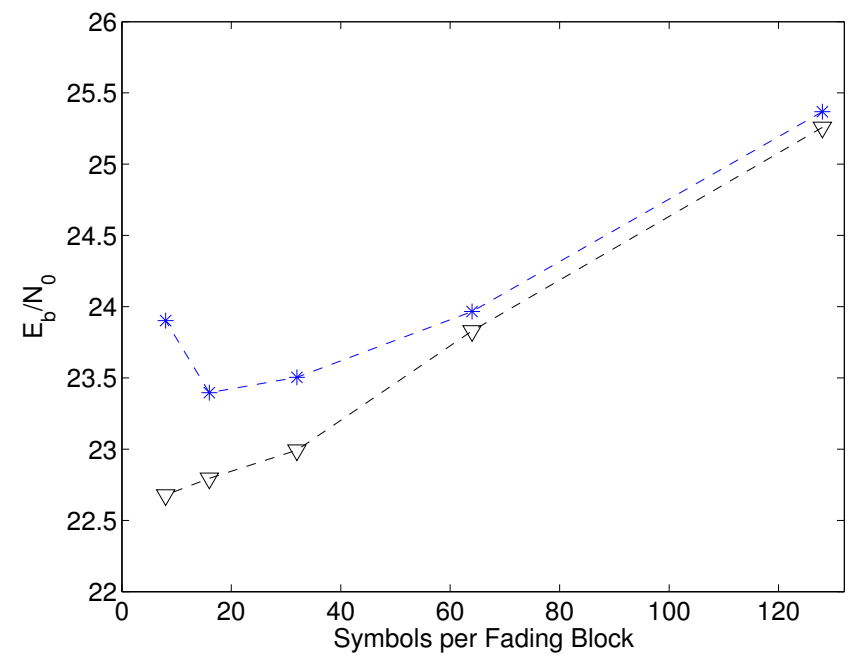

Fig. 5. Signal-to-noise ratio required to reach a bit error rate of $10^{-4}$ at the relay as a function of fading-block length. The curve marked by * denotes the turbo-coded DNC system with estimated fading amplitudes, while the curve marked by $\nabla$ denotes turbo-coded DNC with perfect fading-amplitude knowledge.

margins ranging between 4 and $6 \mathrm{~dB}$. While the LNC system is more energy efficient than the DNC when the same-rate turbo code is used, the throughput of the LNC system is worse than that of the DNC system. The loss in energy efficiency from using DNC versus LNC can be recovered by having the source nodes use a lower-rate turbo code. Consider the performance comparison shown in Fig. 7 for block size $N=32$. DNC at code rate $r_{S}=4500 / 6400$ matches the error-rate performance of LNC at code rate $r_{S}=4500 / 5056$, at $E_{b} / N_{0} \approx 24 \mathrm{~dB}$. At $24 \mathrm{~dB}$, the end-to-end throughput of DNC is higher than LNC, even though DNC uses a lower channel-code rate transmitting to the relay. The throughput gain results from the time slot DNC saves over LNC, as shown in Fig. 1.

To illustrate DNC throughput improvement over LNC, consider the following transmission schedule for the two systems. Assume the end nodes use rate $r_{S}=4500 / 6400$ in DNC, and $r_{S}=4500 / 5056$ in LNC. Assume operation at $E_{b} / N_{0}=24 \mathrm{~dB}$, yielding approximately equal relay error-rate performance. Further, assume that both systems use code rate 


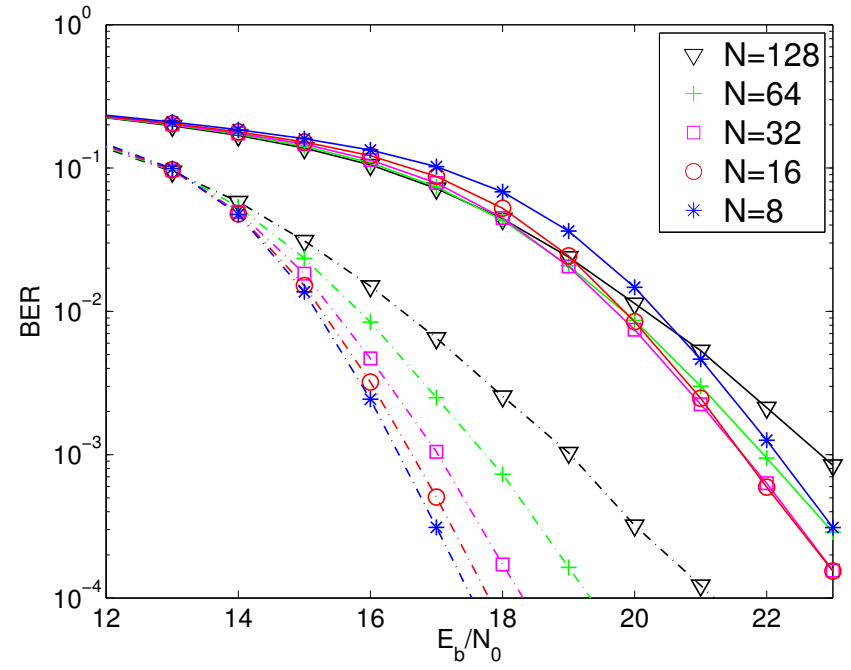

Fig. 6. Comparison of error-rate performance between the turbo-coded DNC and LNC svstems at the relav. DNC - solid lines. LNC - dashed lines.

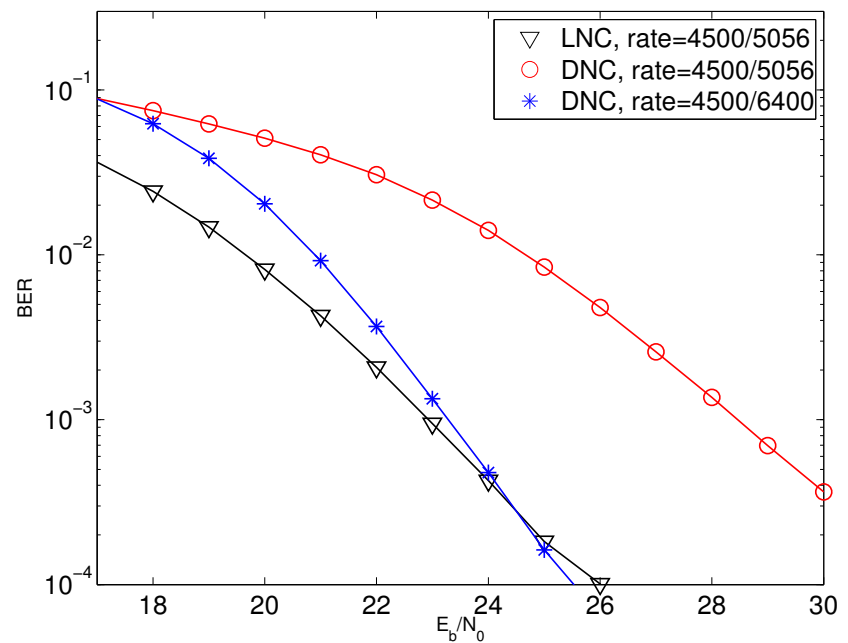

Fig. 7. Comparison of the performance of turbo-coded DNC and LNC at the relay with block size $N=32$. For the DNC system, two code rates are shown, with the lower rate code offering comparable performance to the LNC system.

$r_{R}=4500 / 5056$ for relay broadcast, yielding approximately equal end-to-end performance. DNC requires 6400 channel uses for transmission to the relay versus $2 \times 5056=10112$ for LNC. Both systems require 5056 channel uses for relay broadcast. Throughput for DNC is thus $T^{(D N C)}=9000 /(6400+$ $5056)=9000 / 11,456$ bits per channel use, and for LNC $T^{(L N C)}=9000 /(3 \times 5056)=9000 / 15,168$ bits per channel use. The percentage throughput increase of DNC over LNC is thus $\left(T^{(D N C)} / T^{(L N C)}-1\right) \times 100 \approx 32.4 \%$.

The effectiveness of the transmission-case detector of Subsection IV-A was also investigated through simulation. In particular, simulations were run comparing the performance of DNC both with perfect knowledge of the transmission case and using the transmission-case detector. In both cases, the fading amplitudes were estimated using the estimator of Subsection IV-B. For all block sizes and SNR values, the degradation due to estimating the transmission case was less than $0.1 \mathrm{~dB}$. This small performance gap provides no incentive to refine transmission-case estimates by feeding $a$ priori information back from the turbo decoder, which would also increase the processing delay.

\section{CONCLUSION}

A throughput-improving technique for relaying in the twoway relay network, digital network coding, is refined for practical operation. The system operates noncoherently, providing advantages over coherent operation: there are no requirements for perfect power control, phase synchronism, or estimates of carrier-phase offset.

A computationally simple technique for estimating fading amplitudes at the relay is implemented. Error-rate performance in the noncoherent Rayleigh block-fading channel at several block sizes is presented. The system is simulated with and without an outer error-correcting code. The coded error-rate performance of the system using estimation differs from that with ideal estimates by margins between $0.7-1.5 \mathrm{~dB}$.

When the same-rate turbo code is used, digital network coding has a higher throughput but lower energy-efficiency than link-layer network coding. The energy loss of DNC can be recovered by using a lower-rate turbo code during the MAC phase. Even when the loss of spectral efficiency due to the lower-rate turbo code is taken into account, the DNC system is able to achieve a higher throughput than LNC at the same energy-efficiency. In the particular example presented in this paper, the DNC system is capable of achieving throughputs that are $32 \%$ larger than that of the equivalent LNC system, while operating at the same energy efficiency.

\section{REFERENCES}

[1] R. Ahlswede, N. Cai, S. Li, and R. Yeung, "Network information flow," IEEE Trans. Inform. Theory, vol. 46, pp. 1204-1216, July 2000.

[2] Y. Wu, P. Chou, and S. Kung, "Information exchange in wireless networks with network coding and physical-layer broadcast," in Proc., Conf. Inform. Sci. and Systems (CISS), Baltimore, MD, Mar. 2005.

[3] S. Zhang, S. C. Liew, and P. P. Lam, "Physical-layer network coding," in Proc. MobiComm, 2006, pp. 358-365.

[4] S. Zhang and S. C. Liew, "Channel coding and decoding in a relay system operated with physical-layer network coding," IEEE J. Select. Areas Commun., vol. 27, no. 5, pp. 788-789, June 2009.

[5] S. Katti, S. Gollakota, and D. Katabi, "Embracing wireless interference: analog network coding," Proc. ACM SIGCOMM, pp. 397-408, 2007.

[6] Y. Hao, D. Goeckel, Z. Ding, D. Towsley, and K. Leung, "Achievable rates for network coding on the exchange channel," Proc. IEEE Military Commun. Conf., pp. 1-7, Oct. 2007.

[7] S. Zhang, S. C. Liew, and L. Lu, "Physical layer network coding schemes over finite and infinite fields," IEEE Global Telecommun. Conf., pp. 1-6, 2008.

[8] M. C. Valenti, D. Torrieri, and T. Ferrett, "Noncoherent physical-layer network coding using binary CPFSK modulation," Proc. IEEE Military Commun. Conf., Oct. 2009.

[9] F. Gao, R. Zhang, and Y. C. Liang, "On channel estimation for amplifyand-forward two-way relay networks," IEEE Global Telecommun. Conf., pp. 1-5, 2008.

[10] J. Hamkins, "An analytic technique to separate cochannel FM signals," IEEE Trans. Commun., vol. 48, pp. 543-546, April 2000.

[11] S. Hussain and S. Barton, "Noncoherent detection of FSK signals in the presence of oscillator phase noise in an AWGN channel," in IEEE Mobile and Personal Commun. Conf., Dec. 1993, pp. 95 -98.

[12] J. G. Proakis and M. Salehi, Digital Communications, 5th ed. New York, NY: McGraw-Hill, Inc., 2008.

[13] S. Cheng, M. C. Valenti, and D. Torrieri, "Robust iterative noncoherent reception of coded FSK over block fading channels," IEEE Trans. Wireless Commun., vol. 6, pp. 3142-3147, Sept. 2007.

[14] D. Torrieri, S. Cheng, and M. C. Valenti, "Robust frequency hopping for interference and fading channels," IEEE Trans. Wireless Commun., vol. 56, pp. 1343-1351, Aug. 2008.

[15] European Telecommunications Standards Institute, "Universal mobile telecommunications system (UMTS): Multiplexing and channel coding (FDD)," 3GPP TS 25.212 version 7.4.0, June 2006. 